\title{
TAILS OF THE FIRST HITTING TIMES OF LINEAR DIFFUSIONS
}

\author{
By
}

\author{
Yuji KaSAHARA
}

\begin{abstract}
The tail probability of the first hitting time is discussed for linear diffusions. We obtain the decay rates in terms of the spectral functions and the scale functions. The result is a generalization of recent results of Hamana-Matsumoto for Bessel processes.
\end{abstract}

\section{Introduction}

The diffusion process $X=\left(X_{t}\right)_{t \geq 0}$ with the local generator

$$
\mathscr{G}_{\rho}=\frac{1}{2}\left(\frac{d^{2}}{d x^{2}}+\frac{\rho-1}{x} \frac{d}{d x}\right), \quad x>0(\rho \in \mathbf{R})
$$

is called the $\rho$-dimensional Bessel process and

$$
v:=\frac{\rho}{2}-1
$$

is referred to as the order.

Let $\tau_{b}$ be the first hitting (or, the first passage) time to a point $b(>0)$; i.e.,

$$
\tau_{b}:=\inf \left\{t>0 ; X_{t}=b\right\}
$$

with the convention that inf $\varnothing=\infty$. By the general theory of diffusions the Laplace transform $E_{a}\left[e^{-s \tau_{b}}\right]$ can be expressed in terms of the modified Bessel functions (see [2, p. 398]). In this sense the law of $\tau_{b}$ is completely known. However, it might still be of interest to derive more concrete properties of the law of $\tau_{b}$ through $E_{a}\left[e^{-s \tau_{b}}\right]$.

2010 Mathematics Subject Classification: 60J65, 60J60.

This work was supported by JSPS KAKENHI Grant Number 24540110.

Received November 16, 2015.

Revised June 13, 2016. 
From this viewpoint Hamana-Matsumoto ([4]) recently gave a representation formula for the distribution function $P_{a}\left[\tau_{b} \leq x\right]$ and as its application they obtained the following result. (See also [5] for a refinement.)

THEOREM A. Let $0<b<a<\infty$.

(i) If $v>0$, then

$P_{a}\left(\tau_{b}>t\right)=1-\left(\frac{b}{a}\right)^{2 v}+b^{2 v}\left\{1-\left(\frac{b}{a}\right)^{2 v}\right\} \frac{1}{\Gamma(1+v)(2 t)^{v}}+o\left(t^{-v}\right) \quad(t \rightarrow \infty)$.

(ii) If $v=0$, then

$$
P_{a}\left(\tau_{b}>t\right)=\frac{2 \log (a / b)}{\log t}+o(1 / \log t) \quad(t \rightarrow \infty) .
$$

(iii) If $v<0$, then

$$
P_{a}\left(\tau_{b}>t\right)=a^{2|v|}\left\{1-\left(\frac{b}{a}\right)^{2|v|}\right\} \frac{1}{\Gamma(1+|v|)(2 t)^{|v|}}+o\left(t^{-|v|}\right) \quad(t \rightarrow \infty) .
$$

In fact Yamazato ([13]) has already discussed a similar problem for general linear diffusions under the natural scale. So Theorem A except the case (ii) may also be derived from the results of [13] after a little calculus. On this subject we should also mention the resent result of Uchiyama ([12]). He studied the probability density of $\tau_{b}$ and gave the exact asymptotic forms that are valid uniformly in the initial point $a$.

The aim of the present article is to generalize Theorem A for more general linear diffusions. So the problem and some of the results overlap with those of Yamazato ([13]). However, our point of view is a little different from [13], [4], and [12]: (1) We are mainly interested in the question how the infinitesimal orders (such as $t^{-v}, \log t, t^{v}$ ) and the coefficients can be described in terms of principal characteristics of the diffusion; (2) Another difference (or improvement) is that our argument do not need to exclude the case where $v-\frac{1}{2}$ is an integer, which case was somewhat exceptional in [13] and [4]; (3) The case $v=0$ is studied in a general framework; (4) We shall also study the inverse problem.

Our main results will be given in Sections 5 and 6, where we shall see that the infinitesimal order of the decay of $P_{a}\left[t<\tau_{b}<\infty\right](t \rightarrow \infty)$ comes from the spectral function, while the coefficient will be specified by Feller's scale function. We postpone to state the details until Section 5 and here we give only a typical 
example, which will illustrate the outline of the results. Let $X$ be the diffusion associated with

$$
\mathscr{G}=\frac{1}{2}\left(\frac{d^{2}}{d x^{2}}+(\rho-1) \frac{x}{x^{2}+c^{2}} \frac{d}{d x}\right), \quad x>0,
$$

where $c \geq 0$. Then, for $0<b<a<\infty$ we have

$$
P_{a}\left(t<\tau_{b}<\infty\right) \sim \begin{cases}\frac{s(a ; b)}{s(\infty ; b)^{2}} \frac{2^{-v-1}}{v \Gamma(1+v)} t^{-v} & (v>0), \\ s(a ; b) \frac{2}{\log t}, & (v=0), \\ s(a ; b) \frac{2^{v+1}}{\Gamma(1-v)} t^{v}, & (v<0),\end{cases}
$$

where $v=(\rho / 2)-1$ as before and

$$
s(x ; y)=\int_{y}^{x}\left(u^{2}+c^{2}\right)^{-v-(1 / 2)} d u .
$$

This article consists as follows: In Section 2 we prepare some Tauberian theorems for Laplace transform. In Section 3 we quickly review some of the spectral theory. In Sections 4 and 5 we discuss the case of general linear diffusions and prove some results on the tail probability of the first hitting time in terms of Feller's canonical form of the local generator. In Section 6 we study the case of the diffusion with local generator of the form

$$
\mathscr{G}=\frac{1}{2}\left(\frac{d^{2}}{d x^{2}}+V^{\prime}(x) \frac{d}{d x}\right), \quad x>0,
$$

where $V^{\prime}$ is the derivative of an absolutely continuous function $V$.

\section{Preliminaries from Tauberian Theorems}

First we introduce the following notation, which will be used repeatedly throughout the article.

Notation. We denote by $R_{\rho}(\infty)$ the totality of functions varying regularly at infinity with index $\rho$. That is, $f \in R_{\rho}(\infty)$ means that, for some $A>0$, $f:[A, \infty) \rightarrow(0, \infty)$ is a measurable function such that

$$
\lim _{\lambda \rightarrow \infty} \frac{f(\lambda x)}{f(\lambda)}=x^{\rho}, \quad \forall x>0 .
$$


Similarly, $R_{\rho}(0)$ is the totality of functions varying regularly at 0 with index $\rho$ :

$$
\lim _{\lambda \rightarrow+0} \frac{f(\lambda x)}{f(\lambda)}=x^{\rho}, \quad \forall x>0 .
$$

When $\rho=0$, we say 'slowly varying' rather than 'regularly varying'. Note that $f \in R_{\rho}(\infty)$ if and only if $f(x)=x^{\rho} L(x)$ for some slowly varying $L$. For the properties of regularly varying functions we refer to [3] and [1].

Let $\mu(d x)$ be a finite Borel measure on $[0, \infty)$. Its Laplace transform is defined by

$$
f(s)=\int_{[0, \infty)} e^{-s x} \mu(d x), \quad s>0 .
$$

The relationship between the asymptotic behavior of $\mu[x, \infty)$ as $x \rightarrow \infty$ and that of $f(s)$ as $s \rightarrow+0$ is well known when $(f(0)-f(s)) / s$ diverges. But in the sequel we need the case where it may converge. In order to handle such a case we need the following two theorems. The author do not claim that they are new. But since we do not know good references we shall prove them.

THEOREM 2.1. Let $\varphi \in R_{-\beta}(\infty)(\beta>0)$. Then the following two conditions are equivalent for every $n>\beta$.

$$
\begin{gathered}
\mu[x, \infty) \sim \varphi(x) \quad(x \rightarrow \infty) \\
(-1)^{n} \frac{d^{n}}{d s^{n}} f(s) \sim \beta \Gamma(n-\beta) s^{-n} \varphi(1 / s) \quad(s \rightarrow+0)
\end{gathered}
$$

The extreme case of Theorem 2.1 as $\beta \rightarrow+0$ is

THEOREM 2.2. Let $L \in R_{0}(\infty)$. Then the following two conditions are equivalent.

$$
\begin{gathered}
\frac{\mu[x, \lambda x)}{L(x)} \rightarrow \log \lambda \quad(x \rightarrow \infty), \forall \lambda>1 \\
-f^{\prime}(s) \sim(1 / s) L(1 / s) \quad(s \rightarrow+0)
\end{gathered}
$$

Both imply

$$
\mu[x, \infty) \sim L^{\#}(x):=\int_{x}^{\infty} \frac{L(u)}{u} d u \quad(x \rightarrow \infty)
$$


Later, in the proof of the theorem, we shall see that the integrand in (2.5) is integrable when (2.4) holds.

Note that $L^{\#}$ defined in (2.5) varies slowly. Indeed, for every $c>0$,

$$
L^{\#}(c x)=\int_{c x}^{\infty} \frac{L(u)}{u} d u=\int_{x}^{\infty} \frac{L(c u)}{u} d u .
$$

So, since $L(c u) / L(u) \rightarrow 1(u \rightarrow \infty)$, we conclude that

$$
L^{\#}(c x) \sim \int_{x}^{\infty} \frac{L(u)}{u} d u=L^{\#}(x) \quad(x \rightarrow \infty),
$$

namely, $L^{\#} \in R_{0}(\infty)$.

A typical example of Theorem 2.2 is the following:

EXAMPLE 2.1. Let $\alpha>1$ and $C>0$. If $L(x)=(\log x)^{-\alpha}$, then $L^{\#}(x)=$ $(\alpha-1)^{-1}(\log x)^{1-\alpha}$. Therefore, if

$$
-f^{\prime}(s) \sim \frac{C}{s(\log (1 / s))^{\alpha}} \quad(s \rightarrow+0),
$$

then

$$
\mu[x, \infty) \sim \frac{C}{(\alpha-1)(\log x)^{\alpha-1}} \quad(x \rightarrow \infty) .
$$

For the proofs of Theorems 2.1 and 2.2 we prepare a few lemmas. To begin with consider the following function:

$$
U_{\rho}(x)=\int_{0}^{x} u^{\rho} \mu(d x), \quad x \geq 0
$$

so that

$$
\hat{U}_{\rho}(s):=\int_{[0, \infty)} e^{-s x} d U_{\rho}(x)=\int_{0}^{\infty} e^{-s x} x^{\rho} \mu(d x), \quad s>0 .
$$

Lemma 2.1. Let $0<\beta<\rho$ and $L \in R_{0}(\infty)$. Then the following two conditions are equivalent.

$$
\begin{gathered}
\mu[x, \infty) \sim x^{-\beta} L(x) \quad(x \rightarrow \infty) \\
U_{\rho}(x) \sim \frac{\beta}{\rho-\beta} x^{\rho-\beta} L(x) \quad(x \rightarrow \infty)
\end{gathered}
$$


Proof. In fact more general results will be found in Theorem 2 of Feller [3, p. 283]. However, we shall prove it here for the convenience of the reader.

Suppose that $(2.10)$ holds. Then by the definition (2.8) we have

$$
\begin{aligned}
U_{\rho}(x) & =\rho \iint_{0<y<u<x} y^{\rho-1} d y \mu(d u)=\rho \int_{0}^{x}\{\mu[y, \infty)-\mu[x, \infty)\} y^{\rho-1} d y \\
& =\rho \int_{0}^{x} \mu[y, \infty) y^{\rho-1} d y-\mu[x, \infty) x^{\rho} \\
& \sim \frac{\rho}{\rho-\beta} x^{\rho-\beta} L(x)-x^{\rho-\beta} L(x)=\frac{\beta}{\rho-\beta} x^{\rho-\beta} L(x) \quad(x \rightarrow \infty) .
\end{aligned}
$$

So (2.10) implies (2.11).

Similarly, suppose that $(2.11)$ holds. Since $\mu(d y)=y^{-\rho} d U_{\rho}(y)$, we see

$$
\begin{aligned}
\mu[x, \infty) & =\int_{x}^{\infty} y^{-\rho} d U_{\rho}(y) \\
& =\rho \int_{x}^{\infty} U_{\rho}(u) u^{-\rho-1} d u-x^{-\rho} U_{\rho}(x) \\
& \sim \frac{\rho}{\rho-\beta} x^{-\beta} L(x)-\frac{\beta}{\rho-\beta} x^{-\beta} L(x)=x^{-\beta} L(x),
\end{aligned}
$$

proving (2.10).

In Lemma 2.1 we studied the case where $x \rightarrow \infty$. The next lemma deals with the case where $x \rightarrow+0$.

Lemma 2.2. Let $\beta>0, \beta+\rho>0$ and let $L \in R_{0}(0)$. Then the following two conditions are equivalent.

$$
\begin{gathered}
\mu[0, x) \sim x^{\beta} L(x) \quad(x \rightarrow+0) \\
U_{\rho}(x) \sim \frac{\beta}{\rho+\beta} x^{\rho+\beta} L(x) \quad(x \rightarrow+0) .
\end{gathered}
$$

Proof. The proof is essentially the same as that of Lemma 2.1: Suppose that (2.12) holds. Since 


$$
\begin{aligned}
U_{\rho}(x) & =\rho \iint_{0<y<u \leq x} y^{\rho-1} d y \mu(d u)=\int_{0}^{x}\left(\int_{u}^{x} y^{\rho-1} d y\right) \mu(d u) \\
& =\mu[0, x] x^{\rho}-\rho \int_{0}^{x} \mu[0, y) y^{\rho-1} d y \\
& \sim x^{\rho+\beta} L(x)-\frac{\rho}{\rho+\beta} x^{\rho+\beta} L(x)=\frac{\beta}{\rho+\beta} x^{\rho+\beta} L(x),
\end{aligned}
$$

we have (2.13). To show the converse, interchange the role of $U_{\rho}$ and $\mu$.

We are now ready to prove Theorem 2.1.

Proof of Theorem 2.1. Let

$$
U_{n}(x)=\int_{0}^{x} u^{n} \mu(d x), \quad x \geq 0
$$

as in (2.8). By Lemma 2.1, (2.1) is equivalent to

$$
U_{n}(x) \sim \frac{\beta}{n-\beta} x^{n} \varphi(x) \quad(x \rightarrow \infty),
$$

which is, by the usual Tauberian theorem for Laplace transform (see Theorem $\mathrm{C}$ in Appendix), equivalent to

$$
\hat{U}_{n}(s) \sim \frac{\beta}{n-\beta} \Gamma(1+n-\beta) s^{-n} \varphi(1 / s)=\beta \Gamma(n-\beta) s^{-n} \varphi(1 / s) \quad(s \rightarrow+0),
$$

which can be written as (2.2) because

$$
\hat{U}_{n}(s)=\int_{0}^{\infty} e^{-s x} x^{n} \mu(d x)=(-1)^{n} f^{(n)}(s) .
$$

For the proof of Theorem 2.2 we prepare the next lemma, which is the extreme case of Lemma 2.1 as $\beta \rightarrow+0$.

Lemma 2.3. Let $\rho>0$ and $L \in R_{0}(\infty)$. Then the following three conditions are equivalent.

$$
\begin{gathered}
U_{\rho}(x) \sim \frac{1}{\rho} x^{\rho} L(x) \quad(x \rightarrow \infty) \\
\hat{U}_{\rho}(s) \sim \Gamma(\rho) s^{-\rho} L(1 / s) \quad(s \rightarrow+0) \\
\lim _{x \rightarrow \infty} \frac{\mu[x, \lambda x)}{L(x)}=\log \lambda, \quad \forall \lambda>1
\end{gathered}
$$


Proof. The equivalence of the first two is immediate from Karamata Tauberian theorem. In order to see that they are also equivalent to (2.16), notice that

$$
\mu[x, \lambda x)=\int_{x}^{\lambda x} u^{-\rho} d U_{\rho}(u)
$$

Combining this with

$$
u^{-\rho} d U_{\rho}(u)=\rho u^{-\rho-1} U_{\rho}(u) d u+d\left(u^{-\rho} U_{\rho}(u)\right)
$$

we see that

$$
\mu[x, \lambda x)=\rho \int_{x}^{\lambda x} U_{\rho}(u) u^{-\rho-1} d u+\left\{(\lambda x)^{-\rho} U_{\rho}(\lambda x)-x^{-\rho} U_{\rho}(x)\right\} .
$$

So (2.14) implies

$$
\begin{aligned}
\mu[x, \lambda x) & \sim \int_{x}^{\lambda x} L(u) \frac{d u}{u}+\frac{1}{\rho}\{L(\lambda x)-L(x)\} \\
& =\int_{1}^{\lambda} L(x u) \frac{d u}{u}+\frac{1}{\rho}\{L(\lambda x)-L(x)\} .
\end{aligned}
$$

Therefore, it holds that

$$
\frac{\mu[x, \lambda x)}{L(x)}=\int_{1}^{\lambda} \frac{L(x u)}{L(x)} \frac{d u}{u}+\frac{1}{\rho}\left\{\frac{L(\lambda x)}{L(x)}-1\right\} \rightarrow \log \lambda \quad(x \rightarrow \infty) .
$$

Thus (2.14) implies (2.16).

Conversely, suppose that (2.16) holds. Since

$$
U_{\rho}(x)=\rho \int_{0}^{x} \mu[y, x) y^{\rho-1} d y=\rho \int_{0}^{1} \mu[x \xi, x)(x \xi)^{\rho-1} x d \xi
$$

we have

$$
\frac{\rho U_{\rho}(x)}{x^{\rho} L(x)}=\rho^{2} \int_{0}^{1} \frac{\mu[x \xi, x)}{L(x)} \xi^{\rho-1} d \xi \rightarrow \rho^{2} \int_{0}^{1}\left(\log \frac{1}{\xi}\right) \xi^{\rho-1} d \xi=1,
$$

which implies (2.14).

Proof of Theorem 2.2. Since

$$
-f^{\prime}(s)=\hat{U}_{\rho}(s)
$$


with $\rho=1$, the equivalence of the first two is immediate from the equivalence of (2.16) and (2.15) in Lemma 2.3.

Let us next prove the latter half of Theorem 2.2. Suppose that (2.3) holds. Then we have

$$
\frac{\mu\left[e^{n}, e^{n+1}\right)}{L\left(e^{n}\right)} \rightarrow 1 \quad(n \rightarrow \infty)
$$

which implies

$$
\frac{\mu\left[e^{n}, \infty\right)}{\sum_{k=n}^{\infty} L\left(e^{k}\right)} \rightarrow 1 \quad(n \rightarrow \infty) .
$$

Now using the slowly varying property of $L$, we can deduce

$$
\frac{\mu\left[e^{x}, \infty\right)}{\int_{x}^{\infty} L\left(e^{u}\right) d u} \rightarrow 1, \quad(x \rightarrow \infty),
$$

proving (2.5). The above argument also shows that

$$
\int_{e^{x}}^{\infty} \frac{L(u)}{u} d u\left(=\int_{x}^{\infty} L\left(e^{u}\right) d u\right)<\infty,
$$

for sufficietly large $x$.

\section{Preliminaries from Spectral Theory}

By a (Krein) string we mean a function

$$
m:[0,+\infty) \rightarrow[0,+\infty]
$$

which is nondecreasing and right-continuous. We exclude the trivial case where $m$ is a constant.

For a string $m$ let us review a little on the spectral theory of the generalized Sturm-Liouville operator

$$
\mathscr{L}=-\frac{d}{d m(x)} \frac{d}{d x}, \quad 0<x<\ell
$$

where

$$
\ell=\sup \{x ; m(x)<\infty\} \quad(\in(0, \infty]) .
$$


We define $\varphi_{\lambda}(x)$ and $\psi_{\lambda}(x)(0 \leq x<\ell)$, for every $\lambda \in \mathbf{C}$, as the unique solutions of the following integral equations:

$$
\begin{array}{ll}
\varphi_{\lambda}(x)=1-\lambda \int_{-0}^{x}(x-y) \varphi_{\lambda}(x) d m(y), & 0 \leq x<\ell ; \\
\psi_{\lambda}(x)=x-\lambda \int_{-0}^{x}(x-y) \psi_{\lambda}(x) d m(y), & 0 \leq x<\ell .
\end{array}
$$

Later we shall use the fact that $\varphi_{\lambda}(x)$ and $\psi_{\lambda}(x)$ are entire functions of $\lambda$ for every fixed $x \in[0, \ell)$.

The characteristic function of $m$ is defined by

$$
h(s)=\uparrow \lim _{x \uparrow \ell} \frac{\psi_{-s}(x)}{\varphi_{-s}(x)}\left(=\int_{0}^{\ell-0} \frac{d x}{\varphi_{-s}(x)^{2}}\right), \quad s>0 .
$$

Note that $\varphi_{-s}(x) \rightarrow 1$ and $\psi_{-s}(x) \rightarrow x$ as $s \rightarrow+0$, and hence

$$
h(+0)=\ell .
$$

As is well known, for every $s>0$,

$$
u(s ; x):=\varphi_{-s}(x)-\frac{1}{h(s)} \psi_{-s}(x)
$$

is the nonnegative, nonincreasing solution of

$$
\mathscr{L} u(x)=-s u(x), \quad u(0)=1, \quad u(\ell-0)=0 .
$$

(This fact can easily be seen from the definition of $h(s)$.) So the Green kernel (with respect to $d m(x)$ ) is given by

$$
G_{s}(x, y)=G_{s}(y, x)=h(s) \varphi_{-s}(x) u(s ; y), \quad 0 \leq x \leq y<\ell,
$$

and, therefore,

$$
G_{s}(0,0)=h(s), \quad s>0 .
$$

It is also well known that $h(s)$ has the following representation:

$$
h(s)=a+\int_{-0}^{\infty} \frac{d \sigma(\lambda)}{s+\lambda}, \quad s>0,
$$

where

$$
a=\inf \{x>0 ; m(x)>0\}
$$


and $\sigma:(-\infty, \infty) \rightarrow[0, \infty)$ is a right-continuous nondecreasing function vanishing on $(-\infty, 0)$ such that

$$
\int_{-0}^{\infty} \frac{d \sigma(\lambda)}{1+\lambda}<\infty
$$

The function $\sigma$ is called the spectral function.

Notation. When a string $m(x)$ is given, its right-continuous inverse $m^{*}(x)$ $:=m^{-1}(x)$ is called the dual string. We denote by $h^{*}$ the characteristic function of $m^{*}$. Similarly, $\ell^{*}$ and $\sigma^{*}$ are defined in the obvious manner:

$$
\ell^{*}=\sup \left\{x>0 ; m^{*}(x)<\infty\right\} \quad(=\sup \{m(x) ; x>0\})
$$

and

$$
h^{*}(s)=a^{*}+\int_{-0}^{\infty} \frac{d \sigma^{*}(\lambda)}{s+\lambda}, \quad s>0
$$

Also throughout we define $\hat{h}(s)=1 / h(s)$ and denote

$$
h^{(n)}(s)=\frac{d^{n}}{d s^{n}} h(s), \quad \hat{h}^{(n)}(s)=\frac{d^{n}}{d s^{n}} \hat{h}(s) .
$$

The well-known relationship between $h^{*}$ and $h$ is

$$
h^{*}(s)=\frac{1}{\operatorname{sh}(s)}, \quad s>0
$$

(See e.g. [11]). So it holds that

$$
\hat{h}(s)=s h^{*}(s),
$$

and therefore differentiating the both sides, we see

$$
\hat{h}^{\prime}(s)=h^{*}(s)+s\left(h^{*}(s)\right)^{\prime}=\int_{0}^{\infty} \frac{d \sigma^{*}(\lambda)}{\lambda+s}-\int_{0}^{\infty} \frac{s d \sigma^{*}(\lambda)}{(\lambda+s)^{2}}=\int_{0}^{\infty} \frac{\lambda d \sigma^{*}(\lambda)}{(\lambda+s)^{2}}
$$

and hence we have the following representation formula:

$$
(-1)^{n-1} \hat{h}^{(n)}(s)=n ! \int_{0}^{\infty} \frac{\lambda d \sigma^{*}(\lambda)}{(\lambda+s)^{n+1}}, \quad n \geq 1 .
$$

For our later use we prepare 
LEMmA 3.1. Let $1 \leq k<n$. If $\left|\hat{h}^{(n)}(s)\right| \rightarrow \infty(s \rightarrow+0)$, then

$$
\hat{h}^{(k)}(s)=o\left(\left|\hat{h}^{(n)}(s)\right|\right) \quad(s \rightarrow+0) .
$$

Proof. First recall (3.4). Since

$$
\int_{0}^{\infty} \frac{d \sigma^{*}(\lambda)}{1+\lambda}<\infty
$$

we have

$$
\sup _{s>0} \int_{1}^{\infty} \frac{\lambda d \sigma^{*}(\lambda)}{(s+\lambda)^{k+1}}=\int_{1}^{\infty} \frac{\lambda d \sigma^{*}(\lambda)}{\lambda^{k+1}} \leq \int_{1}^{\infty} \frac{d \sigma^{*}(\lambda)}{\lambda}<\infty .
$$

So we need only to evaluate

$$
\int_{0}^{1} \frac{\lambda d \sigma^{*}(\lambda)}{(s+\lambda)^{k+1}}
$$

Apply Hölder's inequality to

$$
\int_{0}^{1} \frac{\lambda d \sigma^{*}(\lambda)}{(s+\lambda)^{k+1}}=\int_{0}^{1} \frac{\lambda d \sigma^{*}(\lambda)}{(s+\lambda)^{1-(k / n)} \cdot(s+\lambda)^{(n+1) k / n}}
$$

with $1 / p=1-(k / n)$ and $1 / q=k / n$. Then, we have

$$
\begin{aligned}
\int_{0}^{1} \frac{\lambda d \sigma^{*}(\lambda)}{(s+\lambda)^{k+1}} & \leq\left(\int_{0}^{1} \frac{\lambda d \sigma^{*}(\lambda)}{s+\lambda}\right)^{1-(k / n)}\left(\int_{0}^{1} \frac{\lambda d \sigma^{*}(\lambda)}{(s+\lambda)^{n+1}}\right)^{k / n} \\
& \leq\left(\int_{0}^{1} d \sigma^{*}(\lambda)\right)^{1-(k / n)}\left(\int_{0}^{\infty} \frac{\lambda d \sigma^{*}(\lambda)}{(s+\lambda)^{n+1}}\right)^{k / n} \\
& =\text { const } \times\left|\hat{h}^{(n)}(s)\right|^{k / n}=o\left(\left|\hat{h}^{(n)}(s)\right|\right) \quad(s \rightarrow+0) .
\end{aligned}
$$

The next lemma is Proposition 3.1 of [7]. The proof is based on Lemma 3.1 but we omit it and refer to [7].

Lemma 3.2. Suppose $\ell<\infty$ and let $n \geq 1$. If $\left|h^{(n)}(+0)\right|=\infty$ or $\left|\hat{h}^{(n)}(+0)\right|=\infty$, then

$$
\hat{h}^{(n)}(s) \sim-\frac{1}{\ell^{2}} h^{(n)}(s) \quad(s \rightarrow+0) .
$$


Proposition 3.1. Let $n \geq 1$ and suppose that $\varphi \in R_{\beta}(0) \quad(0<\beta<n)$. Then the following two conditions are equivalent.

$$
\begin{gathered}
\sigma^{*}(s) \sim \varphi(s) \quad(s \rightarrow+0) \\
(-1)^{n-1} \hat{h}^{(n)}(s) \sim \beta \Gamma(n-\beta) \Gamma(1+\beta) s^{-n} \varphi(s) \quad(s \rightarrow+0)
\end{gathered}
$$

Proof. By Lemma 2.2, (3.5) is equivalent to

$$
\int_{0}^{\lambda} \xi d \sigma^{*}(\xi) \sim \frac{\beta}{1+\beta} \varphi(\lambda) \lambda \quad(\lambda \rightarrow+0)
$$

which is also equivalent to

$$
\int_{0}^{\infty} \frac{\lambda d \sigma^{*}(\lambda)}{(\lambda+s)^{n+1}} \sim \frac{\beta \Gamma(n-\beta) \Gamma(1+\beta)}{n !} \varphi(s) s^{-n} \quad(s \rightarrow+0)
$$

by Tauberian theorem for Stieltjes transform (apply Theorem $\mathrm{C}$ in Appendix with $\alpha=\beta+1$ ). Keeping (3.4) in mind we see that (3.7) may be rewritten as (3.6).

\section{First Hitting Time of the Diffusion $\frac{d}{d m(x)} \frac{d}{d x}$}

Let $m:[0, \infty) \rightarrow[0, \infty]$ be a string in the sense of Section 3 and let $X=\left(X_{t}\right)_{t \geq 0}$ be a generalized diffusion associated with

$$
\mathscr{G}=-\mathscr{L}:=\frac{d}{d m(x)} \frac{d}{d x}, \quad 0 \leq x<\ell
$$

with reflecting boundary condition at 0 and absorbing boundary condition at $\ell$ when $\ell+m(\ell-0)<\infty$. The state space is $\operatorname{Supp}\{d m\}$. As is well known, $X$ is recurrent if and only if $\ell=\infty$ and is positive recurrent if and only if $m(+\infty)<\infty$.

Let $b \in \operatorname{Supp}\{d m\}$ and let $\tau_{b}$ be the first hitting time of $b$;

$$
\tau_{b}=\inf \left\{t>0 ; X_{t}=b\right\}
$$

as before and we are interested in the decay rate of $P_{a}\left(\tau_{b}>t\right)$ as $t \rightarrow \infty$.

The main tool is the following well-known formula:

$$
E_{x}\left[e^{-s \tau_{b}}\right]=\frac{u(s ; x)}{u(s ; b)} \quad 0 \leq b<x<\ell
$$


where $u(s ; x)$ is defined in (3.2). Note that, if $b=0$, then we have $u(s ; b)=1$ and therefore,

$$
E_{x}\left[e^{-s \tau_{0}}\right]=u(s ; x)=\varphi_{-s}(x)-\frac{1}{h(s)} \psi_{-s}(x), \quad 0<x<\ell,
$$

namely,

$$
E_{a}\left[e^{-s \tau_{0}}\right]=\varphi_{-s}(a)-\hat{h}(s) \psi_{-s}(a), \quad s>0 .
$$

An easy consequence of this formula is the following: Since $\varphi_{-s}(x) \rightarrow 1$ and $\psi_{-s}(x) \rightarrow x, h(s) \rightarrow \ell$ as $s \rightarrow+0$, letting $s \rightarrow+0$ in (4.2), we deduce the wellknown formula

$$
P_{x}\left(\tau_{0}<\infty\right)=1-\frac{x}{\ell}, \quad 0<x<\ell
$$

or, equivalently,

$$
P_{x}\left(\tau_{0}=\infty\right)=\frac{x}{\ell}, \quad 0<x<\ell
$$

(with the convention that $1 / \infty=0)$. Considering the translated string $m_{b}(x):=$ $m(x+b)-m(b)(x \geq 0)$, we have

$$
P_{x}\left(\tau_{b}=\infty\right)=\frac{x-b}{\ell-b}, \quad 0 \leq b<x<\ell(\leq \infty) .
$$

We next evaluate $P_{a}\left(t<\tau_{b}<\infty\right)$ as $t \rightarrow \infty$. By Theorem 2.1 the problem is reduced to the study of $\frac{d^{n}}{d s^{n}} E_{a}\left[e^{-s \tau_{b}}\right]$. So we need to study the $n$-th derivative of (4.3). Since $\varphi_{\lambda}(a)$ and $\psi_{\lambda}(a)$ are entire functions of $\lambda$, we see that, for every fixed $n>1$,

$$
\frac{d^{n}}{d s^{n}} \varphi_{-s}(a) \quad \text { and } \quad \frac{d^{n}}{d s^{n}} \psi_{-s}(a)
$$

remain bounded as $s \rightarrow+0$. So the first term of the right-hand side of (4.3) plays no role. As for the derivatives of the last term of the right-hand side of (4.3), we have

$$
\frac{d^{n}}{d s^{n}}\left(\hat{h}(s) \psi_{-s}(a)\right)=\hat{h}^{(n)}(s) \psi_{-s}(a)+\sum_{k=0}^{n-1}{ }_{n} C_{k} \hat{h}^{(k)}(s) \times O(1) .
$$

Therefore, keeping $\psi_{-s}(a) \rightarrow a(s \rightarrow+0)$ in mind, we can deduce the next result from (4.6) and Lemma 3.1. 
Proposition 4.1. Let $n \geq 1$. If $\left|\hat{h}^{(n)}(+0)\right|=\infty$, then

$$
\frac{d^{n}}{d s^{n}} E_{a}\left[e^{-s \tau_{0}}\right] \sim-\hat{h}^{(n)}(s) a \quad(s \rightarrow+0) .
$$

In what follows $\sigma^{*}$ denotes the spectral function of the dual string $m^{*}(x)=$ $m^{-1}(x)$ as before.

THEOREM 4.1 (recurrent case). Let $0 \leq b<a<\ell$ and let $\varphi \in R_{\beta}(0)(\beta>0)$. Then,

$$
P_{a}\left(\tau_{b}>t\right) \sim(a-b) \Gamma(1+\beta) \varphi(1 / t) \quad(t \rightarrow \infty)
$$

if and only if

$$
\sigma^{*}(\lambda) \sim \varphi(\lambda) \quad(\lambda \rightarrow+0)
$$

Before we proceed to the proof we explain the reason why Theorem 4.1 corresponds to the recurrent case (i.e., $\ell=\infty$ ). The condition (4.7) implicitly claims $P_{a}\left(\tau_{b}=\infty\right)=0$ so that $\ell=\infty$ (see (4.5)). Also (4.8) implies $\sigma^{*}(+0)=0$ so that $s h^{*}(s) \rightarrow \sigma^{*}(+0)=0$ and therefore $\ell(=h(+0))=\infty$.

Proof of TheOrem 4.1. To begin with we remark that the problem is reduced to the case $b=0$. To see this fact consider the translated string

$$
m_{b}(x)=m(x+b)-m(b), \quad x \geq 0
$$

and let $\sigma_{b}^{*}$ be the spectral function of the dual string $m_{b}^{*}$ of $m_{b}$. Since the asymptotic behavior of the spectral function around 0 depends only on that of $m^{*}$ as $x \rightarrow \ell^{*}$ (see [10] and [6]), we know that $\sigma^{*}(\lambda) \sim \sigma_{b}^{*}(\lambda)$ as $\lambda \rightarrow+0$ provided that $\sigma^{*} \in R_{\beta}(0)$ for some $\beta>0$. Thus it is sufficient to consider the case $b=0$ only.

By Proposition 3.1, (4.8) is equivalent to

$$
(-1)^{n-1} \hat{h}^{(n)}(s) \sim \beta \Gamma(n-\beta) \Gamma(1+\beta) s^{-n} \varphi(s) \quad(s \rightarrow+0) .
$$

Since

$$
-\frac{d^{n}}{d s^{n}} E_{a}\left[e^{-s \tau_{0}}\right] \sim a \hat{h}^{(n)}(s) \quad(s \rightarrow+0),
$$


by Proposition 4.1, (4.9) may be written as

$$
(-1)^{n} \frac{d^{n}}{d s^{n}} E_{a}\left[e^{-s \tau_{0}}\right] \sim a \beta \Gamma(n-\beta) \Gamma(1+\beta) s^{-n} \varphi(1 / s) \quad(s \rightarrow+0) .
$$

Now by Theorem 2.1, (4.10) is equivalent to (4.7) (when $b=0$ ).

In Theorem 4.1 the assumption is stated in terms of $\sigma^{*}$, but when $0<\alpha<1$, it may also be written in terms of $\sigma$, because the relationship between $\sigma^{*}$ and $\sigma$ is the following;

Lemma 4.1. Let $0<\alpha<1$. Then, $\sigma \in R_{\alpha}(0)$ if and only if $\sigma^{*} \in R_{1-\alpha}(0)$. Both imply

$$
\sigma^{*}(\lambda) \sim \frac{1}{\Gamma(\alpha) \Gamma(1+\alpha) \Gamma(1-\alpha) \Gamma(2-\alpha)} \frac{\lambda}{\sigma(\lambda)} \quad(\lambda \rightarrow+0) .
$$

This is just an easy consequence of (3.3) and the Tauberian theorem. So we omit the proof.

By Lemma 4.1, Theorem 4.1 may be written as

THEOREM 4.2 (null recurrent case). Suppose that $\sigma \in R_{\alpha}(0)(0<\alpha<1)$ so that $\ell=\infty$. Then, for $0 \leq b<a<\infty$,

$$
P_{a}\left(\tau_{b}>t\right) \sim \frac{a-b}{\Gamma(\alpha) \Gamma(1+\alpha) \Gamma(1-\alpha)} \frac{1}{t \sigma(1 / t)} \quad(t \rightarrow \infty) .
$$

We next study the case where $\sigma \in R_{\alpha}(0)(\alpha>1)$. In this case it holds that $\ell(=h(+0))<\infty$ so that the process is transient.

THEOREM 4.3 (transient case). Suppose that $\ell<\infty$ and $\psi \in R_{\alpha}(0) \quad(\alpha>1)$. Then, for $0 \leq b<a<\ell$,

$$
P_{a}\left(\tau_{b}=\infty\right)=\frac{a-b}{\ell-b}
$$

Furthermore,

$$
\sigma(\lambda) \sim \psi(\lambda) \quad(\lambda \rightarrow+0)
$$

if and only if

$$
P_{a}\left(t<\tau_{b}<\infty\right) \sim \frac{a-b}{(\ell-b)^{2}} \frac{\Gamma(\alpha+1)}{\alpha-1} t \psi(1 / t) \quad(t \rightarrow \infty) .
$$


Proof. For the proof of (4.12) see (4.5). The proof of the latter half can be carried out as that of Theorem 4.1: As we explained before, we may assume that $b=0$. Now assume (4.13) and choose an $n>\alpha$. Then by Tauberian theorem (see Theorem C in Appendix), (4.13) is equivalent to

$$
(-1)^{n} h^{(n)}(s) \sim \Gamma(n+1-\alpha) \Gamma(1+\alpha) s^{-n-1} \psi(s) \quad(s \rightarrow+0) .
$$

By Lemma 3.2, (4.15) is equivalent to

$$
(-1)^{n-1} \hat{h}^{(n)}(s) \sim \frac{1}{\ell^{2}} \Gamma(n+1-\alpha) \Gamma(1+\alpha) s^{-n-1} \psi(s) \quad(s \rightarrow+0),
$$

and by Proposition 4.1 this can be written as

$$
(-1)^{n} \frac{d^{n}}{d s^{n}} E_{a}\left[e^{-s \tau_{0}}\right] \sim \frac{a}{\ell^{2}} \Gamma(n+1-\alpha) \Gamma(\alpha+1) s^{-n-1} \psi(s) .
$$

Applying Theorem 2.1 with $\beta=\alpha-1$ and $\varphi(1 / s)=\frac{a}{\ell^{2}} \frac{\Gamma(\alpha+1)}{\alpha-1} s^{-1} \psi(s)$, we obtain the assertion.

We next turn to the case $\sigma \in R_{\alpha}(0)$ with $\alpha=1$. Note that

$$
\ell=h(+0)=\int_{-0}^{\infty} \frac{d \sigma(\lambda)}{\lambda}=\int_{-0}^{\infty} \frac{\sigma(\lambda)}{\lambda^{2}} d \lambda .
$$

So both $\ell=\infty$ and $\ell<\infty$ are possible under the assumption $\sigma \in R_{1}(0)$. For an example of the case $\ell=\infty$, see Example 2.1.

Theorem 4.4 (critical case). Let $0 \leq b<a<\ell$. Suppose that $\sigma \in R_{1}(0)$ and define $L(x)$ and $L^{\#}(x)$ by

$$
L(1 / s)= \begin{cases}\frac{\sigma(s)}{s}(\ell-b)^{-2} & (\ell<\infty), \\ \frac{\sigma(s)}{s}\left(\int_{s}^{\infty} \sigma(u) u^{-2} d u\right)^{-2} & (\ell=\infty),\end{cases}
$$

and

$$
L^{\#}(x)=\int_{x}^{\infty} \frac{L(u)}{u} d u .
$$

Then $L(x)$ and $L^{\#}(x)$ are slowly varying at $\infty$ and it holds that

$$
P_{b}\left(t<\tau_{a}<\infty\right) \sim(a-b) L^{\#}(t) \quad(t \rightarrow \infty) .
$$


Proof of Theorem 4.4. It is almost trivial that $L(x)$ is slowly varying and we omit the details. For the proof that $L^{\#}(x)$ varies slowly see the comment after Theorem 2.2. So we shall prove (4.19) only.

Since this case is only the extreme case of Theorem 4.1 as $\beta \rightarrow+0$, the proof can be carried out in a similar way. The only difference is that we use Theorem 2.2 in place of Theorem 2.1: When $\ell<\infty$, then $h(+0)=\ell$ provided that $b=0$. (When $b>0$ we need to consider the translated string and hence $h(+0)=\ell-b$ if $\ell$ corresponds to the original $m$.)

By the Tauberian theorem the condition $\sigma \in R_{1}(0)$ implies

$$
-h^{\prime}(s) \sim \sigma(s) s^{-2} \quad(s \rightarrow+0)
$$

and hence, as $s \rightarrow+0$,

$$
h(s) \sim \begin{cases}\int_{s}^{\infty} \sigma(u) u^{-2} d u & (\ell=\infty) \\ \ell-b & (\ell<\infty) .\end{cases}
$$

(Precisely speaking, in the case $\ell=\infty$ we used the fact that $h(\infty)=0$, which can be verified as follows: In general $h(\infty)=\inf \{x>0 ; m(b+x)-m(b-0)>0\}$ but in the present case the right-hand side vanishes because $b$ should be chosen from the support of $d m$.)

Now (4.20) and (4.21) imply

$$
\hat{h}^{\prime}(s)=\frac{-h^{\prime}(s)}{h(s)^{2}} \sim \sigma(s) s^{-2} /\left(\int_{s}^{\infty} \sigma(u) u^{-2} d u\right)^{2} \quad(s \rightarrow+0)
$$

when $\ell=\infty$. The case where $\ell<\infty$ can be shown in a similar way.

Next recalling the definition (4.17) of $L,(4.22)$ may be written as

$$
\hat{h}^{\prime}(s) \sim \frac{1}{s} L\left(\frac{1}{s}\right) \quad(s \rightarrow+0) .
$$

On the other hand we have

$$
\frac{d}{d s} E_{a}\left[e^{-s \tau_{0}}\right] \sim \frac{\partial u(s ; a)}{\partial s} \sim-\hat{h}^{\prime}(s) a \quad(s \rightarrow+0)
$$

as before (see Proposition 4.1). So combining the above two we have

$$
-\frac{d}{d s} E_{a}\left[e^{-s \tau_{0}}\right] \sim a s^{-1} L\left(\frac{1}{s}\right) \quad(s \rightarrow+0) .
$$

So by Theorem 2.2 we have the assertion. 


\section{First Hitting Time of the Diffusion $D_{m} D_{s}^{+}$}

We next study the case of diffusions on an interval $I=(c, r) \subset \mathbf{R}$ with local generator with Feller's canonical form

$$
\mathscr{G}=D_{m} D_{s}^{+}:=\frac{d}{d m(x)} \frac{d}{d s(x)}, \quad x \in I,
$$

where $s(x)$, the scale function, is a strictly increasing continuous function and $d m$, the speed measure, is a positive Radon measure on $I$. (Here, $D_{s}^{+}$denotes the right-derivative with respect to the scale function $s(x)$.)

This case can easily be reduced to the case we studied in the previous section by changing the scale. Indeed, the local generator of $\tilde{X}(t):=s(X(t))$ is

$$
\frac{d}{d \tilde{m}(x)} \frac{d}{d x}, \quad s(c+0)<x<s(r-0)
$$

where $\tilde{m}(x)=m\left(s^{-1}(x)\right)$, and the first hitting time of $X(t)$ to $b$ corresponds to that of $\tilde{X}(t)(=s(X(t)))$ to $s(b)$. We need not to care about the left boundary, because the destination $b(<a)$ should be chosen from the regular point and the law of the first hitting time does not depend on the values of $m(x)$ and $s(x)$ for $x<b$. So in what follows, the spectral function $\sigma$ means that of (5.2) restricted on $\left[s\left(b^{\prime}\right), s(\infty)\right)$ for some regular point $b^{\prime}(\leq b)$ with reflecting boundary condition there. The asymptotic behavior of the spectral function $\sigma$ does not depend on the choice of $b^{\prime}$ (as long as $\sigma$ is regularly varying). So, for example, in the case of Bessel process, we consider only on $[\epsilon, \infty)(\epsilon>0)$. We stress that this understanding is crucial when $\rho \leq 0$. (Otherwise the 'spectral function' does not make sense in general.) Since $s(x)$ allows linear transform, we may and do assume that $s\left(b^{\prime}\right)=0$ without generality.

With the understanding above, let $\sigma(\lambda)$ and $\sigma^{*}(\lambda)$ be the spectral functions of the string $\tilde{m}(x)=m\left(s^{-1}(x)\right)$ and its dual $\tilde{m}^{*}(x):=\tilde{m}^{-1}(x)=s\left(m^{-1}(x)\right)$, respectively. Notice that, if $\sigma$ corresponds to (5.1), then $\sigma^{*}$ corresponds to

$$
\mathscr{G}^{*}:=\frac{d}{d s(x)} \frac{d}{d m(x)} .
$$

Let $a, b(b<a)$ be regular points in the state space $I=(c, r)$, then from Theorem 4.2 we immediately have

THEOREM 5.1 (null recurrent case). If $\sigma \in R_{\alpha}(0)(0<\alpha<1)$, then

$$
P_{a}\left(\tau_{b}>t\right) \sim \frac{s(a)-s(b)}{\Gamma(\alpha) \Gamma(1+\alpha) \Gamma(1-\alpha)} \frac{1}{t \sigma(1 / t)} \quad(t \rightarrow \infty) .
$$


Similarly, we have

THEOREM 5.2 (transient case). If $\sigma \in R_{\alpha}(0)(\alpha>1)$, then

$$
P_{a}\left(\tau_{b}=\infty\right)=\frac{s(a)-s(b)}{s(r-0)-s(b)}
$$

and

$$
P_{a}\left(t<\tau_{b}<\infty\right) \sim \frac{s(a)-s(b)}{(s(r-0)-s(b))^{2}} \frac{\Gamma(\alpha+1)}{\alpha-1} t \sigma(1 / t) \quad(t \rightarrow \infty) .
$$

THEOREM 5.3 (critical case). Suppose that $\sigma \in R_{\alpha}(0)(\alpha=1)$.

(i) If $s(r-0)<\infty$, then (5.4) holds and

$$
P_{a}\left(t<\tau_{b}<\infty\right) \sim \frac{s(a)-s(b)}{(s(r-0)-s(b))^{2}} \int_{0}^{1 / t} \frac{\sigma(u)}{u^{2}} d u \quad(t \rightarrow \infty) .
$$

(ii) If $s(r-0)=\infty$, then,

$$
P_{a}\left(\tau_{b}>t\right) \sim(s(a)-s(b)) L^{\#}(t) \quad(t \rightarrow \infty),
$$

where

$$
L^{\#}(x)=\int_{x}^{\infty} \frac{L(u)}{u} d u \text { where } L(1 / u)=\frac{\sigma(u)}{u} /\left(\int_{u}^{\infty} \sigma(v) v^{-2} d v\right)^{2}
$$

REMARK 5.1. In (5.1), $s(x)$ and $m(x)$ are not uniquely determined by $\mathscr{G}$; i.e., if we replace $s(x)$ and $d m(x)$ by $A s(x)+B$ and $(1 / A) d m(x)$, respectively, then we have the same $\mathscr{G}$. However, under such a transformation, $\sigma$ (or $\sigma^{*}$ ) will be changed to $A \sigma$ (or $\left.(1 / A) \sigma^{*}\right)$ simultaneously and hence the right-hand sides of the results in Theorems 5.1-5.4 do not depend on the choice of $s(x)$.

A typical example of Theorem 5.3 is the following: If $\sigma(\lambda) \sim C \lambda(\log 1 / \lambda)^{\beta}$, then, it is easy to see that, as $x \rightarrow \infty$,

$$
L(1 / s) \sim \begin{cases}\frac{(\beta+1)^{2}}{C}(\log (1 / s))^{-\beta-2}, & (\beta+1>0), \\ \frac{C}{\ell^{2}}(\log (1 / s))^{\beta}, & (\beta+1<0),\end{cases}
$$

and therefore, 


$$
L^{\#}(x) \sim \begin{cases}\frac{\beta+1}{C}(\log x)^{-(\beta+1)}, & (\beta+1>0), \\ \frac{-C}{\ell^{2}(\beta+1)}(\log x)^{\beta+1}, & (\beta+1<0)\end{cases}
$$

(cf. Example 2.1). Thus we have

Corollary 5.1. Let $\beta \neq-1$ and $C>0$. If $\sigma(s) \sim C s\{\log (1 / s)\}^{\beta}(s \rightarrow+0)$, then, for $c<b<a<r$,

$$
P_{a}\left(\tau_{b}=\infty\right)= \begin{cases}0, & (\beta>-1), \\ \frac{s(a)-s(b)}{s(r-0)-s(b)}, & (\beta<-1)\end{cases}
$$

and

$$
P_{a}\left(t<\tau_{b}<\infty\right) \sim \begin{cases}(s(a)-s(b)) \frac{\beta+1}{C}(\log t)^{-(\beta+1)}, & (\beta>-1), \\ \frac{s(a)-s(b)}{(s(r-0)-s(b))^{2}} \frac{-C}{\beta+1}(\log t)^{\beta+1}, & (\beta<-1) .\end{cases}
$$

As a special case $\beta=0$ we have the following: If $\sigma(s) \sim C s(s \rightarrow+0)$, then,

$$
P_{a}\left(\tau_{b}>t\right) \sim(s(a)-s(b)) \frac{1}{C \log t} \quad(t \rightarrow \infty) .
$$

The above three theorems do not include the case of positive recurrent diffusions. But next theorem, which follows immediately from Theorem 4.1, is also applicable to positive recurrent diffusions.

THEOREM 5.4 (recurrent case). If $\sigma^{*} \in R_{\beta}(0)(\beta>0)$, then,

$$
P_{a}\left(\tau_{b}>t\right) \sim(s(a)-s(b)) \Gamma(1+\beta) \sigma^{*}(1 / t) \quad(t \rightarrow \infty) .
$$

\section{Diffusions with Bessel-like Drifts}

In the previous section we studied the case of general linear diffusions. Now let us apply the results to the following special case.

Let $X=\left(X_{t}\right)_{t \geq 0}$ be a diffusion on $I=[0, \infty)$ with local generator of the form

$$
\mathscr{G}=-\mathscr{L}=\frac{1}{2}\left(\frac{d^{2}}{d x^{2}}+V^{\prime}(x) \frac{d}{d x}\right), \quad x>0,
$$


$V(x)$ being an absolutely continuous function on $(0, \infty)$. The Bessel process corresponds to $V(x)=(\rho-1) \log x$.

Put $W(x)=C \exp V(x)$ so that $W^{\prime}(x) / W(x)=V^{\prime}(x)(C>0$ is arbitrary). Then (6.1) may be written as

$$
\mathscr{G}=\frac{1}{2 W(x)} \frac{d}{d x}\left(W(x) \frac{d}{d x}\right), \quad x>0 .
$$

Thus Feller's canonical form of $\mathscr{G}$ is the following:

$$
\mathscr{G}=\frac{d}{d m(x)} \frac{d}{d s(x)},
$$

where

$$
m(x):=2 \int_{0}^{x} W(u) d u, \quad s(x)=s\left(x_{0} ; x\right):=\int_{x_{0}}^{x} \frac{d u}{W(u)}, \quad x \geq 0 .
$$

Here, $x_{0} \in(0, \infty)$ is arbitrary. $x_{0}=0$ and $x_{0}=\infty$ are also allowed as long as the integral makes sense. Recall that, as we pointed out in Remark 5.1, this choice of $x_{0}$ does not affect our results.

The relationship between the asymptotic behavior of $W(x)$ and $\sigma$ (or $\sigma^{*}$ ) will be found in the following Theorem B: (i) is an easy consequence of [7, Theorem 4.2] and (ii) is proved in [8, Proposition 5.1].

THEOREM B. Let $W(\cdot) \in R_{\rho-1}(\infty)$.

(i) If $\rho>0$, then

$$
\sigma(\lambda) \sim \frac{1}{2^{(\rho / 2)-1} \rho \Gamma(\rho / 2)^{2}} \frac{\sqrt{\lambda}}{W(1 / \sqrt{\lambda})} \in R_{\rho / 2}(0) .
$$

(ii) If $\rho<2$, then

$$
\sigma^{*}(\lambda) \sim \frac{2^{(\rho / 2)+1}}{(2-\rho) \Gamma(1-(\rho / 2))^{2}} \sqrt{\lambda} W(1 / \sqrt{\lambda}) \in R_{(2-\rho) / 2}(0) .
$$

So, if $W(\cdot) \in R_{\rho-1}(\infty)$, then $\sigma \in R_{\rho / 2}(0)(\rho>0)$ and $\sigma^{*} \in R_{1-(\rho / 2)}(0)(\rho<2)$. (Both are applicable when $0<\rho<2$.) Therefore, we can apply Theorems 5.2 with $\alpha=\rho / 2$ or Theorem 5.4 with $\beta=1-(\rho / 2)$ according as $\rho>2$ or $\rho<2$;

THEOREM 6.1. Suppose that $W(\cdot) \in R_{\rho-1}(\infty)$ and let $0<b<a<\infty$. We put $v=(\rho / 2)-1$ as before. 
(i) If $\rho>2(v>0)$, then

$$
P_{a}\left(t<\tau_{b}<\infty\right) \sim \frac{s(a)-s(b)}{(s(\infty)-s(b))^{2}} \frac{2^{-v-1}}{v \Gamma(1+v)} \frac{\sqrt{t}}{W(\sqrt{t})} \quad(t \rightarrow \infty) .
$$

(ii) If $\rho<2(v<0)$, then

$$
P_{a}\left(\tau_{b}>t\right) \sim(s(a)-s(b)) \frac{2^{v+1}}{\Gamma(-v)} \frac{1}{\sqrt{t}} W(\sqrt{t}) \quad(t \rightarrow \infty) .
$$

REMARK 6.1. A necessary and sufficient condition for $W(\cdot) \in R_{p-1}(\infty)$ is that $V^{\prime}(\cdot)$ is of the form

$$
V^{\prime}(x)=\frac{\rho-1+o(1)}{x}+\eta(x), \quad(x \rightarrow \infty)
$$

for some $\eta(x)$ such that

$$
\exists \lim _{A \rightarrow \infty} \int_{1}^{A} \eta(x) d x \in \mathbf{R}
$$

This is an easy consequence of the canonical representation of a slowly varying function (see [3, p. 282]).

The case $\rho=2$ is rather complicated. So we give here only a typical case:

THEOREM 6.2. Suppose that $W(x) \sim C x(\log x)^{-\beta}(\beta>-1)$. Then,

$$
P_{a}\left(\tau_{b}>t\right) \sim(s(a)-s(b))(\beta+1) 2^{\beta+1} C(\log t)^{-(\beta+1)} \quad(t \rightarrow \infty) .
$$

Proof. By Theorem B $W(x) \sim C x(\log x)^{-\beta}$ implies

$$
\sigma(\lambda) \sim \frac{1}{2} \frac{\sqrt{\lambda}}{W(1 / \sqrt{\lambda})} \sim \frac{1}{2 C}(\log 1 / \sqrt{\lambda})^{\beta}=\frac{1}{2^{\beta+1} C}(\log 1 / \lambda)^{\beta} \quad(\lambda \rightarrow+0) .
$$

So we have the assertion by Corollary 5.1.

Finally, let us see that Theorems 6.1 and 6.2 include Hamana-Matsumoto's result we mentioned in Introduction (Theorem A).

In the case of Bessel process, we may put $W(x)=x^{\rho-1}\left(=x^{2 v+1}\right)$. So, as is well known (see e.g. page 133 of [2]), the scale function is 


$$
s(x)= \begin{cases}s_{\rho}(0 ; x):=\frac{1}{2-\rho} x^{2-\rho}=-\frac{1}{2 v} x^{-2 v} & (\rho<2 ; v<0), \\ s_{\rho}(1 ; x):=\log x & (\rho=2 ; v=0), \\ s_{\rho}(\infty ; x):=-\frac{1}{\rho-2} x^{2-\rho}=-\frac{1}{2 v} x^{-2 v} & (\rho>2 ; v>0) .\end{cases}
$$

Since the condition that $W \in R_{p-1}(\infty)$ is trivial, we can apply Theorems 6.1 and 6.2 to deduce the assertion of Theorem A.

\section{Appendix}

In this section we briefly sum up some results on Tauberian theorems for Laplace transform and Stieltjes transform.

For a nondecreasing, right-continuous function $\sigma:(-\infty, \infty) \rightarrow[0, \infty)$ such that $\sigma(-0)=0$, we define the Laplace-Stieltjes transform and the generalized Stieltjes transform by

$$
\mathscr{F}[\sigma](t):=\int_{[0, \infty)} e^{-t \lambda} d \sigma(\lambda), \quad t>0
$$

and

$$
H_{n}(\sigma ; s):=\int_{[0, \infty)} \frac{d \sigma(\lambda)}{(s+\lambda)^{n+1}}, \quad s>0 \quad(n \geq 0)
$$

provided that the integral converges.

If

$$
\sigma(\lambda)=\frac{A}{\Gamma(1+\alpha)} \lambda^{\alpha}, \quad \lambda>0,
$$

then

$$
\mathscr{F}[\sigma](t)=A t^{-\alpha}, \quad t>0
$$

and

$$
H_{n}(\sigma ; s)=A \frac{\Gamma(n+1-\alpha)}{n !} s^{\alpha-n-1},
$$

provided that $0<\alpha<n+1$.

The well-known Karamata's extension of Hardy-Littlewood Tauberian theorem is that the constant $A$ may be replaced by slowly varying functions: 
Theorem C. Let $0 \leq \alpha<n+1, A \geq 0$, and $L(x) \in R_{0}(\infty)$. Then, the following three conditions are equivalent.

$$
\begin{aligned}
\sigma(\lambda) & \sim \lambda^{\alpha} L(1 / \lambda) \quad(\lambda \rightarrow+0) \\
\mathscr{F}[\sigma](t) & \sim \Gamma(1+\alpha) t^{-\alpha} L(t) \quad(t \rightarrow \infty) \\
H_{n}(\sigma ; s) & \sim \frac{\Gamma(n+1-\alpha) \Gamma(1+\alpha)}{n !} s^{\alpha-n-1} L(1 / s) \quad(s \rightarrow+0)
\end{aligned}
$$

For the proof of the equivalence of the first two see Feller [3, p. 446] and for the other part we refer to $[9$, Appendix] and $[1$, p. 40].

\title{
References
}

[1] N. H. Bingham, C. M. Goldie, and J. L. Teugels: Regular variation, Encyclopedia of Mathematics and its Applications, 27, Cambridge University Press, Cambridge 1987.

[2] A. N. Borodin and P. Salminen: Handbook of Brownian motion-facts and formulae, Second edition, Probability and its Applications, Birkhauser Verlag, Basel 2002.

[3] W. Feller, An introduction to probability theory and its applications, Vol. II, 2nd ed. John Wiley \& Sons, Inc., New York-London-Sydney 1971.

[4] Y. Hamana and H. Matsumoto: The probability distributions of the first hitting times of Bessel processes, Trans. Amer. Math, Soc, 365 (2013), 5237-5257.

[5] Y. Hamana and H. Matsumoto: Asymptotics of the probability distributions of the first hitting times of Bessel processes. Electron. Commun. Probab. 19 (2014), no. 5, 5 pp.

[6] Y. Kasahara, Spectral function of Krein's and Kotani's string in the class $\Gamma$, Proc. Japan Acad. Ser. A Math. Sci. 88 (2012), no. 10, 173-177.

[ 7 ] Y. Kasahara, S. Kotani: Diffusions with Bessel-like drifts, J. Math. Kyoto Univ. 55 (2015) no. 4, 773-797.

[ 8 ] Y. Kasahara, S. Kotani: Tauberian theorem for harmonic mean of Stieltjes transforms and its applications to linear diffusions, Osaka J. Math. Osaka J. Math. 53 (2016), 221-249.

[9] Y. Kasahara, S. Watanabe: Asymptotic behavior of spectral measures of Krein's and Kotani's strings, J. Math. Kyoto Univ. 50 (2010), 623-644.

[10] S. Kotani, Krein's strings whose spectral functions are of polynomial growth. J. Math. Kyoto Univ. 53 (2013), no. 4, 787-814.

[11] S. Kotani and S. Watanabe: Krein's spectral theory of strings and generalized diffusion processes, Functional analysis in Markov processes (Katata/Kyoto, 1981), 235-259, Lecture Notes in Math., 923, Springer, Berlin-New York 1982.

[12] K. Uchiyama, Asymptotics of the densities of the first passage time distributions for Bessel diffusions, Trans. Amer. Math. Soc. 367 (2015), no. 4, 2719-2742.

[13] M. Yamazato: Hitting time distributions of single points for 1-dimensional generalized diffusion processes. Nagoya Math. J. 119 (1990), 143-172.

\author{
Institute of Mathematics \\ University of Tsukuba \\ Tsukuba, Ibaraki 305-8751 \\ Japan \\ E-mail: kasahara@math.tsukuba.ac.jp
}

